\section{VERGLEICHENDE UNTERSUCHUNGEN ÜBER DEN BILIRUBIN- UND GALLENSÄURESTOFFWECHSEL BEIM LEBERGESUNDEN, LEBERKRANKEN UND NEUGEBORENEN*). \\ Von}

Privatdozent Dr. LEPEHNE.

Aus der Medizinischen Universitätsklinik Königsberg.

Neben dem Nachweis des Auftretens von Gallenfarbstoff im Harn hat uns die Methode der quantitativen Bilirubinbestimmung im Blutserum und im Duodenalsaft nach HiJMANS VAN DEN BERGH einen genaueren Einblick in den Bilirubinstoffwechsel beim Lebergesunden und Leberkranken ermöglicht. Eine weitere Vervollständigung wäre durch eine Methode zur quantitativen Bestimmung der im Urin ausgeschiedenen Gallenfarbstoffmengen $\mathrm{zu}$ erreichen. Wie mir eigene Beobachtungen zeigten, versagt hier die HrJMANS VAN DEN BERGHsche Methode, da die erhaltenen Farbtöne selbst an verdünntem Urin wechselnd sind und von der Farbe der Vergleichsflüssigkeit wesentlich abweichen. Schlechter bestellt steht es um unsere Kenntnisse des Gallensäurestoffwechsels.

Hier macht sich der Mangel an einfachen Methoden zur quantitativen Bestimmung der im Duodenalsaft bzw. im Blut und Urin auftretenden Gallensäuren bemerkbar. Die Petrenkozersche Probe ist am Urin nur unter umständlicher Isolierung der Gallensäuren nach HOPPE-SEYLER u. a. brauchbar. Zu Untersuchungen des Gallensäurengehaltes des Duodenalsaftes đürfte sie auch ungeeignet sein; jedoch habe ich keine Angaben darüber finden können. Für die Bestimmung der Gallensäuren im Blut haben Gilbert, Chabrol und Benard ${ }^{1}$ ) die Pettenkofhrsche Probe verwandt, indem sie Serum mit Alkohol fällten, filtrierten, eindampften, mit Schwefelsäure und Furfurol versetzten und die Gallensäuren spektrophotometrisch bestimmten. Nachprüfungen dieser Methode habe ich in der deutschen Literatur nichtgefunden. Nur von MÜLLER ${ }^{2}$ ) ist neuerdings ebenfalls die Pettenkofersche Probe, und zwar zu einer colorimetrischen Bestimmung der Gallensäuren im Blutserum verwandt worden, ohne daß aber nähere Angaben über die Technik gemacht werden. In den meisten Arbeiten über die Gallensäurenausscheidungen ist eine indirekte Methode angewandt worden. Es wurde die Eigenschaft der Gallensäuren, die Oberflächenspannung herabzusetzen, zum qualitativen und quantitativen Nachweis derselben benutzt. Viefache Untersuchungen hatten gezeigt, da $B$ kaum eine andere überhaupt in Frage kommende Substanz ebenso stark die Oberflächenspannung herabsetzte wie gerade die Gallensäuren. So hatte zuerst HAY die Schwefelblumenprobe zum Nachweis der im Urin ausgeschiedenen Gallensäuren benutzt, die darauf beruht, daß die feinen Körnchen der Schwefelblumen in gallensäurehaltigem Urin infolge der Herabsetzung der Oberflächenspannung zu Boden sinken, während sie auf gallensäurefreiem Urin auf der Oberfläche schwimmen. Ferner wurden stalagmometrische Untersuchungen des Urins und des Blutes vorgenommen. Besonders die französische Schule hat sich viel mit solchen Arbeiten beschäftigt (LYON CAEN, BRULÉ, LEMIERRE u. a.). Neuerdings berichten CHABROL und BÉNARD ${ }^{3}$ ) über derartige Untersuchungen am Urin von Leberkranken. Sie nehmen an, daß eine Herabsetzung der Oberflächenspannung unter 850 fast regelmäBig für Cholurie und Leberschädigung spräche. Für das Blut lehnen sie die Methode $a b$, da die Ausschläge zu geringe seien. Von deutscher Seite liegen einige Bestimmungen der Tropfengröße mittels Stagonometer von JoËL $\left.{ }^{4}\right)$ vor, und zwar sowohl am Urin wie am Serum Leberkranker. Ferner berichteten RETZLAFF ${ }^{27}$ ) und neuerdings BORCHARDT ${ }^{5}$ ) über viscostagonometrische Beobachtungen am Urin und Serum bei Kranken mit Ikterus und bei experimentellem CholedochusverschluB. Auch EpPINGER ${ }^{6}$ ) hat eine kleine $Z$ ahI von Oberflächenspannungsbestimmungen am Urin Ikterischer vorgenommen. Die HAYsche Schwefelblumenmethode wurde nun in letzter Zeit durch MÜLLER ${ }^{11}$ ) einer ausgedehnten Kontrollprüfung unterzogen, die zeigte, daß außer Gallensäuren nur Aminosäuren für den positiven Ausfall der Probe in Frage kämen. Auf Grund dieser Untersuchungen und eigner Kontrollen hatte ich nun versucht, die Schwetelbhumenprobe zu einer annähernden quantitativen Abschätzung der Gallensäureausscheidung im Duodenalsaft und im Urin $z u$ verwenden $^{7}$ ). Ich verdünnte die zu untersuchende Flüssigkeit so lange, bis die in kleinen Schälchen angestellte Schwefelblumen probe negativ ausfiel. Die so erhaltene Verdünnungszahl nannte ich "Gallensäurezahl". Bereits in meiner ersten Veröffentlichung

*) Zum Teil vorgetragen in Wiesbađen am 34. KongreB f. innere Med. betonte ich, daß diese Probe nur klinische brauchbare Vergleichswerte liefern sollte, aber keinen Anspruch auf eine quantitative Untersuchungsmethode mache. Auf dem letzten Kongreß für innere Medizin hat nun ScHADE den Einwand erhoben, da $B$ die Oberflächenspannung zu quantitativen Bestimmungen nicht verwendbar sei. Auf briefliche Anfrage teilte mir Prof. SCHADE mit, daß seiner Ansicht nach bei allen Oberflächenspannungsbestimmungen die Úbertragung in das Gebiet des Quantitativen große Bedenken habe, da es sich um unübersehbare Adsorptionserscheinungen handle. Es werde nur die an der Oberfläche adsorbierte und nicht die im Lösungsraum befindliche Gallensäuremenge gemessen. Auch Jö̈I betont, da $B$ die Kurve der Herabsetzung der Oberflächenspannung bei höherer Konzentration einen asymptotischen Verlauf aufweise, also eine Adsorptionskurve sei (vgl. auch die von $\mathrm{BETH}^{9}$ ) erhaltene Kurve). Aus diesen Gründen darf natürlich die Schwefelblumenmethode nur als eine grob abschätzende und nicht als eine quantitative angesehen werden, die uns infolge ihrer einfachen Technik so lange als klinische orientierende Probe dienen soll, bis eine einfache exakte Gallensäurebestimmungsmethode gefunden ist. Auch $\mathrm{BeTH}^{9}$ ) hat die Messung der Oberflächenspannung des Duodenalsaftes zur quantitativen Bestimmung der ausgeschiedenen Gallensäuren verwandt. Seine Methode dürfte zwar infolge der Isolierung der Gallensäuren und der Verwendung des Stagnometers exakter sein, ist jedoch für rein klinische Zwecke zu kompliziert.

Die beim Lebergesunden mittels meiner Technik erhaltenen Gallensäurezahlen der durch Duodenalsondierung nüchtern gegewonnenen, hellgoldgelben sogenannten , Lebergalle"liegen zwischen $\mathrm{I}: \mathrm{I} 5 \mathrm{O}$ und $\mathrm{r}: 500$ bei einem Gallenfarbstoffgehalt von 3 bis 9 Bilirubineinheiten nach HIJMANS VAN DEN BERGH, wie meine früheren Untersuchungen ${ }^{10}$ ) gezeigt hatten. Ein Parallelgehen der Bilirubin - und Gallensäurezahlen konnte ich nicht feststellen. Höhere Zahlen finden sich in der nach intraduodenaler Magnesiumsulfatinjektion oder Peptoninjektion erhaltenen dunklen sogenannten "Blasengalle". Die Zahlen lagen zwischen I: 500 und I : 2500, wobei den höheren Werten auch hohe Zahlen in der zuerst gewonnenen Lebergalle entsprechen. Während bisher die dunkle Galle als eine Mischung von Gallenblasengalle und Lebergalle infolge reflektorischer Kontraktion der Gallenblase angesehen wurde, setzt sich EINHORN ${ }^{11}$ ) in letzter Zeit mehrfach dafür ein, daß es sich um die Sekretion einer konzentrierteren Lebergalle handle infolge des von dem Darm ausgehenden Reizes. Tatsächlich scheinen. die Verhältnisse komplizierter zu liegen als es bisher von STEPP ${ }^{12}$ ) u. a. angenommen war. Bei meinen früheren Versuchen, mittels der Chromocholie der Frage nach der Herkunft der ,Blasengalle“ näher zu kommen, zeigte es sich, daß in einigen Fällen nach Magnesiumsulfat in der dunklen Galle nicht nur das Bilirubin, sondern auch das ausgeschiedene Indigocarmin entsprechend konzentrierter war. In anderen Beobachtungen hatte sich nur eine Konzentration des Gallenfarbstoffs eingestellt, wie man es von einer $Z$ umischung von Gallenblasengalle, die in der kurzen Zeit nach der Indigocarmininjektion noch keinen blauen Farbstoff enthalten konnte, erwarten mußte. Auch der Vergleich der Gallensäurezahlen und der Bilirubinzahlen der "Blasengalle" deutete in einer Reihe von Untersuchungen auf die Möglichkeit hin, vielleicht zwei verschiedene Quellen für die ,Blasengalle" anzunehmen. Entweder wurden nämlich bei mäBigem Anstieg des Bilirubingehaltes sehr hohe Gallensäurezahlen gefunden - z.B. Bil. 49 Einheiten - Gallensäurezahl $x: I_{400}$ - oder verhältnismäßig niedrige Gallensäurezahl bei besonders starkem Bilirubinanstieg - z. B. Bil. $55^{2}$ Einheiten - Gallensäurezahl I : 450. Nun ergaben mehrfache Untersuchungen bei der Sektion gewonnener Gallenblasengalle ebenfalls niedrige Gallensäurezahlen bei tiefdunkler Farbe (I : 90 bis I : I000, im Mittelwert I : 360 ). Ohne aus diesen und den oben erwähnten noch zu wenig zahlreichen Befunden zu weitgehende Schlüsse ziehen zu wollen, muß doch die Frage nach der Herkunft der ,Blasengalle" noch als nicht ganz geklärt angesehen werden.

Im Urin werden beim Lebergesunden Gallensäuren nicht angetroffen. $\mathrm{Ob}$ im Blut der physiologischen Bilirubinämie auch ein physiologisches Vorkommen von Gallensäuren entspricht, läßt sich wegen der mangelnden Methodik nicht beantworten. 
Wie verhalten sich die Bilirubin- und Gallensäurezahlen beim Leberkranken mit Ikterus? Auf der Höhe des Icterus catarrhalis finden wir, wie bereits mitgeteilt, eine wesentliche Herabsetzung der Gallensäurezahl der "Lebergalle", weniger ausgesprochen der ,,Blasengalle“. Beim Abklingen der Erkrankung steigen die Werte allmählich wieder zur Norm an, wie die in den Tabellen (s. u.) angeführten Zahlen zeigen. Daß diese Ergebnisse der Schwefelblumenmethode den tatsächlichen Verhältnissen entsprechen dürften, geht aus den Beobachtungen von $\mathrm{CHABROL}_{\mathrm{H}}$ und BÉNARD hervor, die gelegentlich eines operativen Eingriffs in der 24stündigen Galle nur einige Gramme Gallensäuren gegenüber $10-20 \mathrm{~g}$ in der 24 Stundenmenge des Gesunden fanden. Auch BETH beobachtete mit seiner Technik beim Icterus catarrhalis eine herabgesetzte Gallensäurenausscheidung im Duodenalsaft. Daß meine Resultate etwa durch Schleimbeimengung vorgetäuscht waren, ist unwahrscheinlich, da Versuche mit Natrium glycocholicum und Zusatz von Mucilago gummi arabici zeigten, daß erst sehr große Mengen von Schleim die Herabsetzung der Oberflächenspannung verhindern und da ein besonders starker Schleimgehalt in den Duodenalsäften keineswegs nachweisbar war.

Die erhaltenen Gallensäurezahien sind unabhängig von dem Gallenfarbstoffgehalt des Duodenalsaftes der Ikteruskranken. Während ich in den früher von mir untersuchten Ikterusfällen auf der Höhe der Gelbsucht meist seh 1 niedrige Bilirubinzahlen erhielt ${ }^{10}$ ), waren jetzt die Zahlen normal (siehe Tabelle Fall L: 6, I Einheiten) oder sogar erhöht (siehe Fall M: 16,25 Einheiten, Fall P: 30 Einheiten). Es bestand also trotz starker Bilirubinämie von $\mathrm{I} 2,25$ bzw. $x_{4}, 8$ Einheiten, die sich durch den prompten Ausfall der direkten Diazoreaktion als eine Bilirubinämie durch Gallenstauung oder durch Resorption von Galle aus den erkrankten Gallencapillaren erwies, eine beträchtliche Pleiochromie. Eine sichere Erklärung dieser Erscheinung läßt sich nicht geben. Es mag sich um erhöhte hepatocelluläre oder anhepatocelluläre Bilirubinbildung handeln. Jedenfalls ist von einem katarrhalischen Verschluß größerer Gallengänge nicht die Rede, wenn man nicht annehmen will, daß ein Teil der Leber durch Gallenstauung versperrt ist und der andere vicariierend verstärkt arbeitet. Weitere Untersuchungen in dieser Richtung würden zur Klärung der Frage des sogenannten katarrhalischen Ikterus, der sicher keine Einheit ist, beitragen. Auch STRIsower ${ }^{13}$ ) beobachtete 3 Fälle von Icterus catarrhalis mit normalen Bilirubinwerten des Duodenalsaftes bei hohen Blutbilirubinzahlen, ohne eine stichhaltige Erklärung beibringen zu können.

Mit dem Ablilingen der Gelbsucht steigen die Bilimbinzahlen des Duodenalsaftes an, da es zu einer Ausscheidung des im. Blut und im Gewebe angehäuften Farbstoffes kommt, was man auch "sekundäre Pleiochromie" nennen könnte (siehe Fall L und M). Entsprechend sinkt der Gehalt des Blutes an Gallenfarbstoff sukzessive zur Norm, wie es die Tabellen deutlich erkennen lassen; die Bilirubinurie verschwindet und macht stärkerer Urobilinogenurie Platz.

Gegenüber diesen klaren Verhältnissen des Bilirubinstoffwechsels können wir über das Verhalten der Gallensäuren im Blut nichts Sicheres sagen.

Nach den Untersuchungen von CHABROL und BENARD mittels der PeTtENKoferschen Probe soll die in Blute der Ikteruskranken befindliche Gallensäuremenge eine überaus geringe sein. Die Oberflächenspannung des Serums ist, wie die Beobachtungen von Jö̈L, RETZLAFF und von BORCHARDT zeigen, auch nur in geringem Grade herabgesetzt, um beim Abklingen der Erkrankung wieder anzusteigen. Die Oberflächenspannung des Blutserums wird aber durch Gallensäuren in viel geringerem Grade herabgesetzt als die des Urins oder Duodenalsaftes. Erst nach Zusatz von beträchtlichen Mengen von Natrium glycocholicum zu Blutserum beginnt, wie eigene Versuche zeigten, die Schwefelblumen probe positiv zu werden. $\mathrm{ES}_{\mathrm{S}}$ ist daher die Bestimmung der Oberflächenspannung für das Blutserum auch nur zu einer annähernden quantitativen $\mathrm{Ab}$ schätzung der in ihm befindlichen Gallensäuremengen unbrauchbar. Auch CHABROL und BENARD lehnen diese Untersuchungsmethode $a b$, wie ich es bereits erwähnt habe.
Zur weiteren Klarstellung des Gallensäurestoffwechsels bleibt daher vorläufig nur die Untersuchung des Urins übrig. Dabei zeigten meine Beobachtungen, daßim Urin selbst auf der Höhe der Gelbsucht anscheinend nur ganz geringe Mengen von Gallensäuren ausgeschieden wurden, da die Schwefelblumenprobe nur bis $\mathrm{zu}$ einer Verdünnung von höchstens I : 3 oft sogar nur am unverdünnten Urin ( $\mathrm{I}: 0)$ positiv ausfiel. $Z u$ dem gleichen Resultat waren CHABRol und BÉNARD sowohl mittels Bestimmung der Tropfengröße, sowie auf chemischem Wege gekommen, so daß von einem Parallelgehen der Gallenfarbstoff- und Gallensäurenausscheidung, wie es BORCHARDT behauptet, wenigstens quantitativ nicht die Rede sein kann. Die niedrigen Zahlen sind durch die Methode an sich nicht bedingt. Im Gegenteil konnte ich mehrfach nachweisen, daß Lösungen von Natrium glycocholicum in Urin höhere Verdünnungszahlen ergaben als gleich konzentrierte Lösungen in Wasser, was wohl auf dem die Oberflächenspannung weiter herabsetzenden Gehalt an Salzen zurückzuführen ist [siehe auch JoËI ${ }^{4}$ ) und DouMer ${ }^{14}$ )]. So fand ich bei Na. glyc. in Wasser gelöst die Verdünnungszahl I : 40oo, bei Na. glyc. im Urin eines Gesunden I : $I_{5}$ Ooo. CHABrol und BÉnARD ziehen aus ihren Befunden den Schluß, daß die Bildung der Gallensäuren während des Ikterus wesentiich herabgesetzt sei. Auch BЕтн kommt zu dieser Schlußfolgerung, die auch schon von älteren Autoren auf Grund chemischer Analysen der Galle beim Stauungsikterus des Menschen angenommen worden war [siche NAUNYN $\left.{ }^{32}\right)$ ]. Diese Frage scheint mir aber solange nicht beantwortbar zu sein, als uns eine gute Methode zum quantitativen Nachweis der Gallensäuren im Blut fehlt. Denn es könnte sich auch um einen Ubertritt der in der Leber in normaler Menge gebildeten Gallensäuren ins Blut handeln, in dem sie gebunden werden und daher weder in größerer Menge im Urin erscheinen, noch mit den derzeitigen Methoden genügend im Blut nachweisbar sind.

f. Wenn CHABRol und BÉNARD als Gegenbeweis anführen, daß bei einem Hunde nach intravenöser Injektion von Natriumglycocholat dasselbe sofort im Urin auftrat, so beweist dies wohl nichts für den Menschen, da ja beim Hunde auch für das Bilirubin der Nierenschwellenwert im Gegensatz zum Menschen ein sehr niedriger ist [Hijmans van DeN Bergh, Rosenthal und Meyer ${ }^{15}$ )]. Ferner zeigten PONDER ${ }^{11}$ ), daß er bei einem Kaninchen nach i. $v$. Injelution von $20 \mathrm{ccm}$ einer Natriumtaurocholatlösung von I ; 80einer Menge, die übrigens ohne Anwesenheit von Serum genügen würde, um I\% aller Erythrocyten des Tieres in 7 Minuten zur Lösung zu bringen - im Urin mittels Isolierung und PETTENKoFerscher Reaktion nur Spuren von Gallensäuren nachweisen konnte. Über den Nierenschwellenwert der Gallensäurenausscheidung beim Menschen können wir also entgegen der hypothetischen Annahme BORCriardTs nichts Sicheres sagen.

Was die Dauer der Gallensäurenausscheidung im Urin im Vergleich zur Gallenfarbstoffausscheidung betrifft, so stehen meine Erfahrungen im Gegensatz zu denen MÜLLERs ${ }^{17}$ ), der Gallensäuren im Urin beim Icterus catarrhalis auch noch nach dem Verschwinden des Gallenfarbstoffs nachweisen konnte. Ich benutzte zu meinen Untersuchungen ursprünglich Sulfur sublimatum*), das MüLlER als die empfindlichste Schwefelblüte bezeichnete. Weitere Versuche zeigten mir aber, daß Sulfur depuratum meist auf höhere Verdünnungen ansprach als Sulfur sublimatum (z. B. Na. glycocholicumlösung mit Sulfur sublimatum positiv bis I : 4000 mit Sulfur depuratum bis fast I : Io ooo). Aber selbst mit diesem Präparat und bei einer Beobachtungszeit von 30 Minuten war es mir nicht möglich, nach Aufhören der Bilirubinausscheidung noch Gallensäuren regelmäßig nachzuweisen. Auch BorCHARDT gibt an, daß die Messung der Tropfengröße nach Aufhören der Bilirubinurie wieder normale Werte ergebe. Wie die Tabelle des Falles P, zeigt, fehlte der positive Ausfall der Schwefelblumenprobe sogat noch zur Zeit starker Bilirubinurie, was allerdings a uf einer gewissen Unempfindlichkeit der Schwefelblumenprobe (BORCHARDT) beruhen mag.

In den nun folgenden Tabellen sind die systematischen, von Woche $\mathrm{zu}$ Woche durchgeführten Untersuchungen der Leber-

*) Nicht Sulfur praecipitatum, wie es versehentlich hieB. 
galle und Blasengalle auf Gallensäuren und Gallenfarbstoff, des Urins auch auf Urobilinogen und des Blutes auf Bilirubingehalt und Ablauf der direkteri Diazoreaktion von 3 Fällen von Icterus catarrhalis niedergelegt, die die oben geschilderten Verhältnisse klar erkennen lassen.

Bemerkenswert ist das Verhalten der direkten Diazoreaktion, die mit dem Abklingen des Ikterus vom prompten Typus über den zweiphasig-prompten und zweiphasig-verzögerten zum rein verzögerten Typus bei noch erhöhten Blutbilirubinwerten verläuft. Diese bekannte Erscheinung läßt daran denken, daß es beim Icterus catarrhalis auch zu einer Vermehrung des sogenannten , funktionellen Bilirubins" im Blute kommt, die sich aber erst beim Verschwinden des ,Stauungsbilirubin"-Anteils aus dem Blute manifestiert. Thannhausers ${ }^{18}$ ) Angabe, $d a \beta$ sich "Staunngsbilimbin" mit prompter direkter Diazoreaktion durch Stehenlassen in ,funktionelles Bilirubin" mit verzögerter direkter Reaktion umwandle, habe ich mehrfach nachgeprït, aber nicht im Sinne Thannhausers bestätigen können. Größere Serummengen eines Stauungsikterus kann man tagelang stehen lassen, ohne daß die rite ausgeführte direkte Diazoreaktion (siehe Erg. d. inn. Med. u. Kinderheilk. 20, 236) ihren prompten Charakter irgendwie ändert. Läßt man kleine Serummengen mit geringem Gehalt an Stauungsbilirubin in kleinen Reagensgläschen offen am Licht stehen, so fällt die Reaktion nach 2 Tagen allerdings mehr oder weniger verzögert, zugleich aber auch im ganzen bedeutend schwächer aus und zwar, meiner Ansicht nach, nicht, weil sich das Stauungsbilirubin in funktionelles Bilirubin umgewandelt hat, sondern weil das leichter oxydierbare Staungsbilirubin inzwischen zu Biliverdin oxydiert ist und somit der prompte Anteil der Reaktion verschwindet, da Biliverdin keine Diazoreaktion gibt. So ist nur das bei jedem Ikterus anscheinend ebenfalls vermehrt im Blut kreisende ,funktionelle Bilirubin" [siehe oben; auch RETZLAFF ${ }^{27}$ ) vertritt diese Ansicht; vg1. auch meine Experimente mit Unterbindung des Ductus choledochus $\left.{ }^{11}\right)$ ], das schwerer oxydierbar ist, zurückgeblieben. Man könnte daran denken diesen Anteil des funktionellen Bilirubins beim Staungsikterus dadurch annähernd abzuschätzen, daß man verschiedene Verdünnungen des Serums ansetzt und bis zum Eintritt verzögerter Reaktion stehen läßt, um dann die Bilirubinmenge $\mathrm{zu}$ bestimmen und mit der Verdünnungszahl zu multiplizieren. Thannhausers Befund, daß eine stärkere Nitritlösung eine verzögerte Reaktion in eine prompte überführe, konnte ich nicht bestätigen. Uber die Ursache der verschiedenen Serumreaktionen sind wir immer noch nicht im klaren. Ich habe früher die Ausdrücke ,funktionelles Bilirubin" und "Stauungsbilirubin“" gebraucht und gebe $\mathrm{zu}$, daß ein Rückschluß auf den Ort der Bilirubinbildung nur als Arbeitshypothese anzusehen ist. Jedenfalls muß man die durch die verschiedenen Reaktionen gekennzeichneten Zustände des Serumbilirubins [prompte und verzögerte Reaktion, verschiedene Oxydierbarkeit, verschiedene Eiweißadsorption, verschiedene Chloroformlöslichkeit nach GRUNENBERG ${ }^{20}$ ), verschiedenes Verhalten bei der Ultrafiltration nach LEschke $\left.{ }^{11}\right)$ ], unterscheiden, ohne damit chemische Unterschiede in molekularen Bau als sicher hinzustellen, was ja BrimanN ${ }^{21}$ ) durch seine spektrophotometrischen Messungen widerlegen zu können glaubt. Neuerdings behaupteten Strauss und AdLER ${ }^{22}$ ), daß der Ausfall der Reaktion von dem Verhältnis des Serumalbumins zum Serumglobulin abhänge.

Bei Leberkranken ohne Ikterus fand sich positive Schwefelblumenprobe bei Lebercarcinommetastasen, bei Cholelithiasis und Lebercirrhose, aber auch hier nur in einem Teil der Fälle und nur bis zu ganz niedrigen Verdünnungszahlen. Bei Stauungsleber und bei Infektionskrankheiten (Ty., Scharl., Tbk.) war die Probe negativ. Somit kann ich MÜllers Ausspruch, daß die Schwefelblumenprobe die empfindlichste Reaktion zum Nachweis der gestörten Leberfunktion sei, was in ähnlicher Form auch schon BRULé, Garban und LE GAL LA

Fall L

\begin{tabular}{|c|c|c|c|c|c|c|c|c|c|}
\hline \multirow{2}{*}{ Datum } & \multirow{2}{*}{ Ilkterus } & \multicolumn{2}{|c|}{ Lebergalle } & \multicolumn{2}{|c|}{ Blasengalle } & \multicolumn{3}{|c|}{ Urin } & \multirow{2}{*}{$\begin{array}{c}\text { Blut- } \\
\text { Bilirubin }\end{array}$} \\
\hline & & Gallensäuren & Bitir. & Gallensäuren & Bilir. & Gallens. & Bilir. & Ug. & \\
\hline 6. III. & mäßig & I : IO & $6, \mathbf{I}$ & - & 一 & $I: O$ & $+t$ & $\begin{array}{l}+ \\
+\end{array}$ & $\begin{array}{c}\mathrm{I} 3, \mathrm{O} \\
\text { prompt } \\
2,8\end{array}$ \\
\hline I4. III. & gering & I $: 80$ & $.13,75$ & I $: 350$ & 27,5 & $I: O$ & - & $\begin{array}{l}+ \\
t\end{array}$ & $\begin{array}{c}\text { zweiphasig } \\
\text { prompt }\end{array}$ \\
\hline
\end{tabular}

Fall M.

\begin{tabular}{|c|c|c|c|c|c|c|c|c|c|}
\hline \multirow{2}{*}{ Datum } & \multirow{2}{*}{ Ikterus } & \multicolumn{2}{|c|}{ Lekergalle } & \multicolumn{2}{|c|}{ Blasengalle } & \multicolumn{3}{|c|}{ Urin } & \multirow{2}{*}{$\begin{array}{l}\text { B'ut- } \\
\text { Bilirub:n }\end{array}$} \\
\hline & & Gallensäuren & B lir. & Gallensäuren & Bilir. & Gallens. & Bilir. & Ug. & \\
\hline 4. III. & mäßig & I $: 30$ & I6,25 & I : IOO & 60 & $I: O$ & + & $\begin{array}{l}t \\
t\end{array}$ & $\begin{array}{c}\text { I } 4,8 \\
\text { prompt }\end{array}$ \\
\hline 8. III. & gering & I : I 50 & 30,5 & I $: 200$ & 90 & 一 & - & $\begin{array}{l}+ \\
+\end{array}$ & $\begin{array}{l}3,5 \\
\text { prompt }\end{array}$ \\
\hline I 5. III. & fast - & $\begin{array}{rrl}\text { I I } & : 170 \\
\text { II } & \text { I } & : 280\end{array}$ & $\begin{array}{l}29,5 \\
26,0\end{array}$ & - & - & - & - & + & $\begin{array}{c}2,5 \\
\text { zweiphasig } \\
\text { verzögert }\end{array}$ \\
\hline
\end{tabular}

Fall P.

\begin{tabular}{|c|c|c|c|c|c|c|c|c|c|}
\hline \multirow{2}{*}{ Datum } & \multirow{2}{*}{ Ikterus } & \multicolumn{2}{|c|}{ Lebergalle } & \multicolumn{2}{|c|}{ Blasengalle } & \multicolumn{3}{|c|}{ Urin } & \multirow{2}{*}{$\begin{array}{c}\text { Blut- } \\
\text { Bilirubjn }\end{array}$} \\
\hline & & Gallensäuren & Biliz: & Galiensäuren & Bilir. & Gallens. & Bilir. & Ug. & \\
\hline I 5. II. & stark & I $: 30$ & 30 & I $: 600$ & 180 & - & $+t$ & $\begin{array}{c}++ \\
+\end{array}$ & $\begin{array}{l}12,25 \\
\text { prompt }\end{array}$ \\
\hline 22. II. & mäßig & $1: 15$ & 24,8 & I $: 300$ & I 30 & - & + & $\begin{array}{c}++ \\
+\end{array}$ & $\begin{array}{c}4.5 \\
\text { prompt }\end{array}$ \\
\hline I. III. & gering & $1: 150$ & 33,5 & I : 500 & I I 4 & - & - & +4 & $\begin{array}{c}2,5 \\
\text { prompt }\end{array}$ \\
\hline 8. III. & gering & $1: 250$ & 40 & $I: 550$ & 57 & - & - & $+t$ & $\begin{array}{c}1,8 \\
\text { zweiphasig } \\
\text { prompt }\end{array}$ \\
\hline I 5. III. & gering & I : 160 & 35,5 & I $: 450$ & $55^{2}$ & - & - & ++ & $\begin{array}{c}2,0 \\
\text { zweiphasig } \\
\text { verzögert }\end{array}$ \\
\hline 22. III. & 0 & I : 300 & 20,25 & I : I 500 & 71 & - & - & + & $\begin{array}{c}0,75 \\
\text { verzögert }\end{array}$ \\
\hline
\end{tabular}

SALLE ${ }^{23}$ ) behaupteten, wenigstens mit den mir zur Verfügung stehenden Schwefelpräparaten nicht anerkennen. Nach BorchaRDT ist übrigens die Haysche Probe der Stalagmometrie bedentend an Empfindlichkeit unterlegen. Bemerkenswert ist der positive Ausfall der Probe (bis $\mathrm{I}: 2$ ) im Urin einer bereits im Abklingen begriffenen Hyperemesis gravidarum, was vielleicht nicht auf Gallensäuren, sondern a uf Aminosäurenausscheidung beruhen dürfte, die ebenfalls die Oberflächenspannung stark herabsetzt und die bei den Graviditätstoxikosen erhöht sein soll. Weitere allerdings wenig zahlreiche Untersuchungen am Urin gesunder Schwangerer in den letzten Monaten der Gravidität ergaben ein negatives Resultat. Crainiciand und PoPPER ${ }^{29}$ ) dagegen wollen unter 47 gesunden Schwangeren in den letzten Monaten der Gravidität in $20 \%$ positive Hay sche Probe und in $8 \%$ sogar Bilirubinurie (?) beobachtet haben. 
Beim Neugeborenen ist der Bilirubinstoffwechsel vielfach weitgehend untersuclit worden. Wenig zahlreich sind die Angaben : über den Gallensäurenstoffwechsel. Duodenalsondierungen, die auf diese Verhältnisse gerichtet waren, liegen bisher kaum vor. Es gelang mir unter ca. 40 Sondierungsversuchen bei Neugeborenen 20mal Duodenalsaft zu erhalten. Dabei zeigte es sich, daß beim Neugeborenen, wie es auch die Stuhluntersuchungen von YLPPo ${ }^{23}$ ) vermuten ließen, in der Regel eine Pleiochromie besteht, und zwar anscheinend unabhängig vom Alter des Säuglings und vom Bestehen oder Nichtbestehen eines Icterus neonatorum. Sechsmal fand ich Werte unter 9 Bilirubineinheiten. $\mathrm{Ob}$ es sich hier um Verdünnung durch Pankreaswerte handelt oder die Werte mitunter tatsächlich niedrige sind, bleibe dahingestellt. In den übrigen Fällen erhielt ich Zahlen von 1o-46 Einheiten, wie ich sie früher beim hämolytischen Ikterus und bei der perniziösen Anämie des Erwachsenen gefunden hatte. Somit dürfte in der Regel eine Störung der Gallenfarbstoff ausscheidenden Funktion der Säuglingsleber nicht bestehen. Die Gallensäurezahlen waren nun im Gegensatz zu diesen hoheñ Bilirubinwerten in der Mehrzahl der untersuchten Fälle mit 1:30 bis $1: 140$ auffallend niedrig und nur wenige Male konnten etwas höhere Werte bis I : $25^{\circ}$ notiert werđen. Ausführlicher habe ich die Ergebnisse der Sondierungen und anderer Untersuchungen, sowie die ganze Frage der Pathogenese des Icterus neonatorum in einer demnächst in der. Festschrift für Herrn Geheimrat Winter (Monatsschrift für Geburtshilfe und Gynäkologie) erscheinenden Arbeit besprochen. Die Leber des Neugeborenen scheint einen Teil der Gallensäuren in das Blut übertreten $\mathrm{zu}$ lassen. Dafür dürfte auch sprechen, daß ich unter 26 untersuchten Urinen von $2-24$ Tage alten Neugeborenen rmal positive Schuefelblumenprobe erhalten konnte. (Vergleiche auch die positiven Gallensäurebefunde im Urin der Säuglinge mit Icterus neonatorum von BiRCH-HiRschFeid, Halberstam, Hofmeister u. a.) Auch hier kann eine Klärung des Gallensäurestoffwechsels aber erst erfolgen, wenn es gelingt, die Gallensäurekurve im Blut ebenso festzulegen wie die Bilirubinkurve. Der Befund von RosenTHAL und NoSSEN ${ }^{24}$ ), daß der Neugeborene in weit stärkerem Maße als bei den meisten Lebererkrankungen des erwachsenen Menschen einen beträchtlichen Mangel an trypanoziden Substanzen in seinem Serum aufweist, könnte vielleicht auch im Sinne des Kreisens von größeren Gallensäurenmengen im Blut gedeutet werden. LANGE ${ }^{25}$ ) glaubt nämlich, daß es sich dabei um eine Zerstörung des Komplements im Blut durch Gallensäuren handle, was zwar Rosenthal ablehnt. Allerdings fanden LIEDTKE und KöRBER ${ }^{11}$ ) bei toluylendiaminund phosphorvergifteten ikterischen Hunden nur geringgradige Schwankungen des Komplementgehaltes.

Die starke, nach der Geburt noch ansteigende Bilirubinämie, die jeder Neugeborene aufweist und die als Grundlage des Icterus neonatorum anzusehen ist, dürfte in Anbetracht der verzögerten Diazoreaktion im Blutserum der ungestörten und pleiochromen Gallenfarbstoffausscheidung in den Darm zumeist auf einer Uberschwemmung des Blutes mit aus der Placenta $\left(\mathrm{SCHICK}{ }^{26}\right.$ ) und dem retikuloendothelialen Zellsystem der Leber und Milz stammendem funktionellem Bilirubin beruhen, der die an sich gut ausscheidenden Leberzellen nicht gewachsen sind.

L it e r a t u r: 1) Gilbert, Chabrol, Bénard, Cpt. rend. des séances de la soc. de biol. 83, Nr. 37. 1920. - ${ }^{2}$ ) MüLler, Schweiz. med; Wochenschr. 1922, Nr. 5. - ${ }^{3}$ ) Chabrol u. BÉnard, Gaz. des hop. civ. et milit. 192 I, Jg. 94, Nr. 28 (Kongreßzentralbl. 21, S. 322. 1922). - ${ }^{4}$ ) JÖ̈L, Biochem. Zeitschr. II9; 93. I92 I. $\left.{ }^{5}\right)$ Borchardt, Klin. Wochenschr. 1922, Nr. 20. - ${ }^{6}$ ) EPPINGER, hepato-lienale Erkr. - 7) MüLler, Schweiz. med. Wochenschr. 1921. Nr. 36. - ${ }^{8}$ ) Lepenne, Münch. med. Wochenschr. I922, Nr. Io. - ${ }^{9}$ ) BETH, Wien. Arch. f. inn. Medizin. 2, 565. I92 I. 10) LEPEHNE, Dtsch. Arch. f. klin. Med. I37, 78. I92 I. - 11) EINHORN, New York med. Journ. (KongreBzentralbl, 2O, S. 453. I92 I) und Arch. f. Verdauungskrankh. Bd. 29, 1922. - 12) STEPP, Zeitschr, f. klin. Med. 87, 313. I920. - ${ }^{13}$ ) Strisower, Wien. Arch. f. inn. Med. 3, 153. 1921. - ${ }^{14}$ ) Doumer, Ann. des méd. I92 I, Nr. I (KongreBzentralbl. 20, 292. I92 I). - ${ }^{15}$ ) ROSENTHAL u. MEYER, Arch. f. exp. Pathol. u. Pharmakol. 91, 246. I921. - ${ }^{16}$ ) Ponder, Brit. journ. of. exp. pathol. 2, 289. I92 I. - (KongreBzentralblatt 23. 25. I922.) - 17) Müller, Schweiz. med. Wochenschr. 1922, Nr. 5. 18) Thannhauser, Dtsch. Arch. f. klin. Med. I37, I79. I921. 19) Lepenne, Dtsch. Arch. f. klin. Med. I36, 88. I921. - ${ }^{20}$ ) GRUNenberg, 34. Kongreß f. inn. Med. $-{ }^{21}$ ) Beckmann, 33. Kongreß f. inn. Med. 192I, S. 48 I. - ${ }^{22}$ ) Strauss u. Adler, 34. Kongreß f. inn. Med. - ${ }^{23}$ ) YLPPO, Zeitschr. f. Kinderheilk. 9, 208. 1913. ${ }^{24}$ ) Rosenthal u. Nossen, Berl. klin. Wochenschr. I92 I, Nr. 37. $\left.{ }^{25}\right)$ LANGE, Klin. Wochenschr. I922, Nr. 21. $-{ }^{26}$ ) ScHICK, Zeitschr. f. Kinderheilk. 27, 231. I92 I. - ${ }^{27}$ ) RetZLAFr, Diskussion Berl. med. Gesellsch. Berl. klin. Wochenschr. 1921, S. 81 I. - ${ }^{28}$ ) Brulk, Garban, Le Gai la Salle, Rev, de chirurg. I9I4, Nr. 6 (Kongreßzentralblatt II, 622. I9I4.). - ${ }^{29}$ ) CRAINICIANU u. Popper, Presse med. I921, Nr.43. (Kongreßzentralblatt I8, 468. I92I). - ${ }^{30}$ ) LÜDKE u. KöRBER, Beiträge zur klin. d. Infektionskrankh. u. z. Immunitätsforsch. I, 473. I9r3. - ${ }^{31}$ ) LEschKE, Diskussion Berl. med. Ges. Berl. klin. Wochenschr. I92 I, 884. - 32) Naunyn, Grenzgebiete 31. 537.1919.

\section{ÜBER DIE SOGENANNTE GRÜNE BENZALDEHYD- REAKTION IM BILIRUBINHARN.}

Von.

Dr. K. Hoesch, Sekundärarzt.

Aus der I. med. Abteilung des städt. Krankenhauses Nürnberg. (Vorstand: Direktor Prof. Dr. JOH. MULLER.)

Die in Nr. 18 der Klinischen Wochenschrift erschienene Arbeit von Meyer-Estorf enthält den Hinweis, daß bei einer Gruppe bestimmter Ikterusformen im Harn auf Zusatz von Ehrlichs Reagens eine intensive Grünfärbung auftritt. Meyer hält die Ansicht Eppingers, daß es das Bilirubin des Harns sei, welches sich bei Zugabe von Ehrlichs Reagens gelegentlich durch Oxydation grün färbe, für unrichtig, weil diese Grünfärbung auch in dem bilirubinfreien Tierkohlefiltrat des gleichen Harns unter der Einwirkung dieses Reagens zustande käme.

Vor kurzem (Nr. 22 dieser Zeitschrift) hat nun in der Tat WELTMANN zeigen kömnen, daß auch der bloße Harnstoff mit dem Dimethylamidobenzaldehyd des Ehrlichschen Reagens unter Entstehung einer grünlichen Färbung reagiert. Die Reaktion war nicht überraschend, da man schon lange wußte, daß der Harnstoff $z u$ verschiedenen Aldehyden eine große Affinität besitzt. Auch wir bemerkten diese Harnstoffaldedydreaktion seit längerer Zeit; nach Zusatz des Reagenses entsteht ein zeisiggelber bis hellgrüner Farbton, der freilich nur dann in Erscheinung tritt, wenn er nicht durch normale oder pathologische Harnfarbstoffe zugedeckt wird. Infolgedessen bekamen wir bei den verschiedenen Ikterusformen die Reaktion im unfiltrierten Harn nur selten, meist nur bei abklingender Gelbsucht oder in der Rekonvaleszenz zu Gesicht, wenn Bilirubin nicht mehr oder in Spuren und auch keine Urobilinogenvermehrung vorhanden und der Harn überdies noch urochromarm war; regelmäßiger tritt sie in hellen normalen Urinen auf.

Vollkommen wesensverschieden aber von der beschriebenen Harnstoffreaktion ist die Grünfärbung, die nicht selten in bilirubinhaltigen Harnen nach Zusatz des Ehrlichschen Reagenses auffällt. Schon rein äußerlich tritt der Gegensatz durch den mehr bläulichen Farbton und die meist bedeutend stärkere Farbintensität hervor. Es konnte aber dann festgestellt werden, daß der Aldehyd des Harns mit dieser Reaktion nichts zu tun hat, sondern $\mathrm{da} \beta$ der hierfür verantwortliche Anteil des Reagenses nur die Salzsäure ist. Reine Chlorwasserstofflösung der gleichen Konzentration hat stets denselben Effelst, wie das vollständige Ehrlich sche Reagens. Wir konnten weiter zeigen, daß bei Zusatz aller anorganischen und organischen Säuren (soweit sie nicht nur spurweise dissoziiert sind) die bald schneller, bald langsamer entstehende Farbe nur ganz schwache Differenzen der Nuance aufweist, welche auf Nebenreaktionen vereinzelter Säuren zurückzuführen sind. Es sind demnach die H-Ionen, welche das Eintreten dieser Färbereaktion bedingen. Bei Verwendung des Ehrlichschen Reagens wird dabei, wie oben erwähnt, die gleichzeitig vor sich gehende- Harnstoff-Aldehydreaktion 